\title{
Promoting adolescent health: insights from developmental and communication neuroscience
}

\author{
RUI PEI \\ Annenberg School for Communication, University of Pennsylvania, Philadelphia, PA, USA \\ ELISSA C. KRANZLER \\ Annenberg School for Communication, University of Pennsylvania, Philadelphia, PA, USA \\ AHNA BALLONOFF SULEIMAN \\ University of California Berkeley, School of Public Health, Berkeley, CA, USA \\ EMILY B. FALK* \\ Annenberg School for Communication, University of Pennsylvania, Philadelphia, PA, USA
}

\begin{abstract}
Adolescence is a period of remarkable psychosocial and neural development. Many life-long health habits are established during adolescence, making it a window of opportunity for health promotion. One way to promote adolescent health is through mass and social media campaigns. Although some health media campaigns that target adolescents are effective in changing health-relevant cognitions and behaviors, there is considerable room for improving these outcomes. Recent advancements combining neuroimaging tools and health persuasion have suggested key neural mechanisms underlying behavior change and retransmission of healthrelevant ideas and norms in adults. This line of work highlights the integral role of the brain's value system in health persuasion and its importance for improving campaign design and effectiveness. Less is known about how these insights could be leveraged to inform adolescent health persuasion. In this article, we review what is known and unknown about the development of the brain's value system and its connections with cognitive control and social cognition systems across adolescence. Combining these insights, we propose that neuroimaging tools offer unique possibilities that could improve adolescent media health campaigns and promote adolescent well-being.
\end{abstract}

Submitted 4 October 2017; revised 31 May 2018; accepted 16 July 2018

\footnotetext{
* Correspondence to: Annenberg School for Communication, University of Pennsylvania, 3620

Walnut Street, Philadelphia, PA 19104, USA. Email: falk@asc.upenn.edu
} 


\section{Introduction}

Adolescence denotes the maturational period that commences with the onset of puberty and ends with the establishment of an independent, adult role in society (Crone \& Dahl, 2012; Blakemore \& Mills, 2014; Steinberg, 2014). Often defined by its age range (which is thought to extend from age nine or ten through the early twenties; Pringle et al., 2016), adolescence represents a sensitive developmental period for learning about and adopting a wide range of health behaviors. The physical, neural, psychological and social changes that begin with puberty contribute to skill learning, exploring novel environments and risk-taking behaviors. Risk-taking during adolescence can promote positive outcomes, such as developing and engaging in new intellectual and artistic ideas, forging new social relationships and becoming increasingly involved in one's community (for a recent review, see Telzer, 2016), as well as negative outcomes, including increased tobacco and drug use (Warren et al., 2000; Dahl, 2004; Grant et al., 2004), risky driving (Williams, 2003; Simons-Morton et al., 2005) and criminal behavior (Zimring, 1998).

Adolescence is a critical period for establishing behavioral patterns (Jessor, 1984). Behaviors established during adolescence, from health-promoting habits such as engaging in physical activity and healthy eating to healthharming behaviors like smoking and drinking, are likely to continue throughout life and have a long-lasting influence on individuals' health and well-being (Kleinert, 2007). From an anthropological perspective, the increased tendency for risk-taking during adolescence is functionally adaptive, as this tendency facilitates the exploration of new social roles, the creation of meaningful relationships, and engagement in other rewarding experiences (Ellis et al., 2012). Nevertheless, adolescents' vulnerability to engaging in harmful risks can lead to negative social and behavioral trajectories. The potential long-term influence of these behaviors underscores the importance of enhancing opportunities for positive, pro-social risk-taking and decreasing engagement in harmful behaviors during this developmental period.

Public health professionals have long recognized the importance of preventing the adoption of unhealthy behaviors during adolescence and have approached this issue, in part, through media campaigns. Mass media health campaigns have been effective health promotion tools for delivering health messages to large groups of people (Hornik, 2002), targeting adolescent health behaviors including smoking initiation (Farrelly et al., 2003; Wakefield et al., 2003; Farrelly et al., 2005), risky sexual behavior (Zimmerman et al., 2011) and marijuana use (Palmgreen et al., 2007). The success of health messages relies critically on an understanding of the psychological processes that underlie persuasion and the various social, environmental and developmental factors 
that may influence this process. Yet, adolescents' exposure to campaign messages does not always produce the intended outcomes (Noar, 2006; Brennan et al., 2012; Aubrey et al., 2014; for a review, see Noar, 2006), suggesting the need for additional variables that can predict message effectiveness.

In the past decade, empirical studies have provided evidence that neuroimaging methods may add additional value to the design and selection of health messages and interventions. Brain signals collected during health message exposure in small groups of people have been found to predict message success at both the individual and population levels and help illuminate the underlying cognitive mechanisms that may lead to persuasion (Falk et al., 2010; Chua et al., 2011; Falk et al., 2011; Falk et al., 2012; Berkman \& Falk, 2013; Wang et al., 2013; Cooper et al., 2015; Falk et al., 2015; Wang et al., 2015; Weber et al., 2015; Falk et al., 2016; Cooper et al., 2017). Across these studies, neural activation in the ventromedial prefrontal cortex (VMPFC) during exposure to health messages has consistently been linked to health behavior change. It has been theorized that neural response in VMPFC during health message exposure signals the assessment of the value of the health message to oneself (Cooper et al., 2015; Falk et al., 2015) or the integration of the message's value with one's self-concept (Vezich et al., 2017). However, the brain's value system undergoes considerable changes during adolescence, influenced by developments in cognitive control and social cognition systems. These developmental considerations may shape the way in which findings from adult studies can be applied to adolescents.

Despite a growing body of literature in social and communication neuroscience, scant research has examined the role of the brain in adolescent health persuasion. Considering that the brain undergoes significant development during puberty and adolescence, the extent to which insights from adult neuroscience studies of persuasion and social influence can be applied to adolescents remains an open question. Combining empirical evidence from the neuroscience of persuasion and the neurodevelopment of the value system during adolescence, we highlight potential opportunities to integrate insights about the adolescent brain in designing messages that channel adolescents' motivations toward pro-health behaviors and creating messages that maximally harness the power of social media.

\section{Adolescent health behavior and media health campaigns}

Adolescence is a dynamic developmental period. The hormonal changes that stimulate adolescent pubertal maturation are accompanied by complex physical, social and emotional changes (Steinberg \& Morris, 2001; Crone \& Dahl, 2012). As noted above, these changes are associated with increased 
risk-taking, which in turn can produce preventable injury and death. According to a 2010 report by the Centers for Disease Control and Prevention (Miniño, 2010), the top three causes of death during adolescence are accidents (most of which are caused by motor vehicle crashes), homicide, and suicide. To reduce the initiation and prevalence of harmful and potentially life-threatening behaviors in adolescents, considerable resources have been invested in health promotion interventions, including health-focused mass media campaigns. Mass media health campaign messages, which range in format from professionally developed public service announcements (PSAs) to entertainment education programs (e.g., TV shows with health messages embedded in the storylines), are disseminated through an array of media platforms, including television, radio and newspapers. These platforms are effective tools for broadcasting health messages to large populations (Noar, 2006). The success of these campaigns is contingent on the content of the messages and audience members' exposure to these messages during routine media use (Wakefield et al., 2010) and is facilitated by social interactions surrounding the messages (Brennan et al., 2016).

To this end, media campaigns can achieve intended cognitive and/or behavioral outcomes through direct and indirect pathways (Wakefield et al., 2010). Many campaigns aim to directly affect individuals by invoking cognitive or emotional responses through direct exposure to campaign messages. For instance, a campaign aimed to prevent smoking onset may highlight specific risks of smoking in campaign messages, with the expectation that individual exposure to these messages will influence adolescents to espouse increased anti-smoking beliefs. Complementary to this approach, campaigns can also achieve intended outcomes through indirect pathways, such as interpersonal communication about campaign messages (Katz \& Lazarsfeld, 1955; Katz, 1957). For example, exposure to messages can prompt individuals to discuss the content of messages with peers, resulting in downstream effects on peers' normative beliefs or behaviors consistent with message content (Brennan et al., 2012). More broadly, health campaigns can prompt changes in public policy at the institutional level by promoting public discussions of the issue (e.g., policy restrictions on the age of tobacco purchase influencing tobacco use), thereby influencing individual behavior (McCombs \& Shaw, 1972; Goldberg et al., 1997; Hornik, 2002; Hornik \& Yanovitzky, 2003). The direct and indirect pathways of influence highlight the importance of optimizing campaigns with respect to at least two sets of campaign outcomes: (1) changes in health-related cognitions and behaviors that result from direct campaign exposure; and (2) the extent to which individuals share and positively discuss campaign messages with others. 
Indirect pathways to campaign effects (e.g., interpersonal sharing and influence) may be amplified by the new media environment. There has been immense growth in adolescent engagement in media activities and use of media devices in recent years (Jordan \& Romer, 2014; Lenhart, 2015; Rideout, 2016). With its rapid increase in popularity, particularly among adolescents, social media is a promising avenue for the dissemination of health campaign messages targeting adolescents. According to a 2015 report from the Pew Research Center, 92\% of adolescents aged 13-17 use the internet daily, and the majority of them $(71 \%)$ use more than one social media platform (Lenhart, 2015). Campaigns targeting adolescents, such as 'truth' and 'The Real Cost' anti-smoking campaigns, increasingly incorporate new media channels into their campaign strategies in addition to conventional media outlets, disseminating campaign messages online through social media platforms such as Facebook and Instagram. This type of media platform provides unique functions (i.e., online sharing and commenting) that may increase campaign reach and engagement (Korda \& Itani, 2013; Rus \& Cameron, 2016). More broadly, online components in a campaign may improve the efficacy of campaign messages, though it should be noted that these functions can also undermine campaign effectiveness if not channeled properly (Geber et al., 2017).

In sum, mass media and social media have strong potential to influence adolescent behavioral trajectories. Below, we argue that neuroscience studies of persuasion can complement existing campaign evaluation studies and laboratory studies of message effects by providing additional information about the mechanisms through which messages influence cognitions and behavior. We then review key ways in which adolescent brains differ from adult brains and suggest ways in which this may impact the optimal design of research and interventions at this intersection.

\section{Neuroscience studies of persuasion in adults}

Current approaches to health intervention design and the prediction of health behaviors make strong use of self-report measures both by asking people to directly evaluate campaign messages (Atkin \& Freimuth, 2001) and by manipulating message features and then measuring people's self-reports of the cognitions that follow (Cappella, 2006). For example, campaign developers often recruit members of the campaign's target audience to rate the effectiveness of campaign advertisements during the message testing phase of development or report the frequency of their exposure to campaign ads as part of a formal evaluation (Atkin \& Freimuth, 2001). Other approaches include systematically varying features of health messages, such as message content and 
form, and subsequently measuring people's behavioral and cognitive reactions to the manipulated features (Cappella, 2006). Neuroimaging tools can complement both of these types of designs by providing insight into the real-time underlying mental processes that occur during message exposure without the need for participant introspection or retrospection about the processes elicited by messages, thereby circumventing some potential biases (Paulhus, 1986; Fazio \& Olson, 2003; Dijksterhuis, 2004) introduced by self-report measures (Lieberman, 2010). Neuroimaging refers to a broad set of techniques for measuring brain activity, such as functional magnetic resonance imaging (fMRI), functional near-infrared spectroscopy and electroencephalography, among others. fMRI, which uses changes in blood flow in the brain as an indicator of neural activity, is among the neuroimaging methods most commonly used to study health persuasion.

In the last decade, a growing number of studies have demonstrated that neural signals during exposure to health messages can predict key campaign outcomes including behavior change and message sharing. These studies generally adopt a 'brain-as-predictor' approach (Berkman \& Falk, 2013), which uses signals in brain systems previously implicated in psychological processes to predict cognitive and behavioral outcomes. Brain response during health message exposure has been shown to predict individual-level health behaviors such as changes in smoking-relevant behaviors (Chua et al., 2011; Falk et al., 2011; Wang et al., 2013; Riddle et al., 2016; Owens et al., 2017), sunscreen use (Falk et al., 2010; Vezich et al., 2017) and physical activity (Falk et al., 2015). Consistent evidence suggests that neural activity in the medial prefrontal cortex (MPFC) can predict message-consistent behaviors, although the specific subregions of the MPFC vary across studies, with some studies reporting dorsal subregions of MPFC, and some reporting more ventral subregions of MPFC (VMPFC) (for reviews, see Cacioppo et al., 2016; Falk \& Scholz, 2018). Within this body of work, several studies have shown that brain responses to persuasive health messages can account for variance in health outcomes above and beyond self-report measures (Falk et al., 2010; Falk et al., 2011; Riddle et al., 2016). In one study (Falk et al., 2011), smokers were shown a series of anti-smoking ads during an fMRI scan. Following each ad, participants rated the extent to which the ad made them feel a sense of self-efficacy regarding quitting smoking, their intention to quit and the self-relevance of the ad. Exhaled carbon monoxide (CO), a biological indicator of smoke exposure, was measured before and one month after the scanning session. Neural activation in the VMPFC during ad exposure was a significant predictor of changes in expired $\mathrm{CO}$ levels, controlling for self-reported intentions to quit, self-efficacy to quit and self-relevance of the ads. The authors found that VMPFC neural activity on average predicted $23 \%$ of the variance in 
smoking behavior change on top of the variance predicted by self-reported attitudes and intentions. A follow-up study that compared model fit between models using self-reported predictors and models using self-report and neural measures reported similar figures ranging from $14 \%$ to $32 \%$ (Tables 5-7 from Riddle et al., 2016), depending on the characteristics of the stimuli and participants. It is worth noting that the number of relevant studies is still small and that future studies that examine the conditions under which neural measures provide additional predictive power compared to self-report or behavioral measures will be useful.

A related brain-as-predictor approach has been used to predict populationlevel message-induced behavior change (Falk et al., 2014; Falk et al., 2016). This approach traverses different levels of analysis in that neural activity in a small group of individuals is used to forecast population-level message success. For instance, neural activation within the VMPFC, a brain region thought to underlie the computation of the value for the self, in small samples of smokers predicted real-world success of smoking cessation messages at the population level (Falk et al., 2012; Falk et al., 2016). This approach has also been employed to predict the population-level success of cultural phenomena, such as song downloads (Berns \& Moore, 2012), microloans raised (Genevsky \& Knutson, 2015) and the success of movies (Boksem \& Smidts, 2014). These initial studies demonstrate that neural signals in the brain's valuation system (VMPFC and ventral striatum [VS]) during exposure to a wide range of stimuli can reflect population-level preferences related to those stimuli. However, the underlying mechanisms that explain these relationships, as well as the boundary conditions under which neural responses do and do not reflect population-level outcomes, have not yet been fully examined.

In addition to predicting direct effects on health behavior change, neural responses to messages can also predict whether people share campaign-relevant information. In this respect, neural responses to messages may also help forecast indirect campaign effects such as what information is likely to spread from person to person. Recent studies have linked neural activity within positive valuation, self-related processing, and social cognition regions during exposure to health-related news items with both individual-level intention to share the news articles (Baek et al., 2017) and total number of shares at the population level (Scholz et al., 2017). The authors demonstrated that neural activity in the brain's self-relevance processing and social cognition networks increases brain activity in the value system, which in turn predicts population-level article virality. This suggests that messages that are more personally relevant for the individual (e.g., mentioning issues that one deeply cares about) or messages that induce more thoughts about social consequences of sharing and/or other people's mental states (e.g., containing information that is considered useful 
for the individual's friends) may lead to greater perceived value of sharing and are thus more likely to be shared. Together, these studies highlight the importance of self-relevant and social cognitions when individuals consider the value of sharing health messages, demonstrating that neural signals in the value system in a small group of people can predict population-level popularity.

Given the accumulating findings that link VMPFC neural activity and message-relevant outcomes, several hypotheses have been proposed to account for why VMPFC activity may be linked to behavior change and message sharing. One hypothesis is that brain signals in the value system indicate an individual's overall computed value of a message to oneself (Cooper et al., 2015; Falk et al., 2015; Vezich et al., 2017). This idea was further developed into a valuation model of influence and message sharing (Falk \& Scholz, 2018), proposing that a common pathway of subjective value maximization underlies people's decisions about sharing information or being influenced by a message. Health messages that engage greater responses in the brain's valuation regions are thought to elicit more thoughts about personal value and are therefore more successful at changing individual health behaviors and being shared with others. This account links social science research on persuasion (Cialdini \& Goldstein, 2004; Berger, 2014; Albarracin \& Shavitt, 2018) with the neuroeconomic decision-making model (Levy \& Glimcher, 2012), which states that activity in the VMPFC and VS is key in computing the subjective value of a wide range of stimuli (Levy \& Glimcher, 2012; Bartra et al., 2013). The valuation model is echoed in prominent theories of behavior change (Fishbein \& Ajzen, 2011), according to which individuals weigh different considerations relevant to a behavior (such as their behavioral beliefs or self-efficacy) when assessing the value of engaging in the behavior, and it expands prior theories by highlighting the significance of self- and social-related inputs.

In sum, studies from social and communication neuroscience demonstrate that neural activity can serve as a significant predictor of health behavior outcomes and health message sharing at both individual and population levels. Current theory suggests that VMPFC and VS activity during message exposure may be an indicator of a valuation process in which one weighs a message's value against one's self-concept and social relevance, and that brain responses in these regions are important predictors of outcomes across a range of studies. There is currently less research on the neural mechanisms underlying persuasion in adolescents, and the extent to which insights learned from adult persuasion research can be extended to adolescents is unclear. Next, we discuss a core set of neural changes in adolescents that are relevant to these questions, with particular emphasis on the value system. 


\section{Developmental changes in brain systems underlying decision-making}

A substantial body of research in developmental neuroscience has laid the groundwork for understanding the normative neurodevelopmental changes that underlie adolescent decision-making. In general, at least two sets of maturational processes influence adolescent decision-making ${ }^{1}$ : (1) increased reward sensitivity in the valuation system, along with increased salience for social and affective influences; and (2) a gradual strengthening of cognitive control, self-inhibition and the ability to align behavior with long-term goals (Suleiman \& Dahl, 2017). The first of these processes is thought to underlie changes in motivation and increased sensitivity to certain types of learning experiences - especially those associated with social relationships. Compared to the second set of processes, which are less dependent on pubertal maturation (Dahl, 2004), the increase in reward sensitivity and social saliency occurs relatively abruptly and is more directly linked with puberty (Braams et al., 2015).

Reward and value processing are associated with activation in similar brain regions in adolescents and adults, including the VMPFC and the VS. These regions are responsive to primary rewards such as food and sex, as well as more abstract forms of rewards such as the well-being of others and smiling faces (Bjork et al., 2004; May et al., 2004; Ernst et al., 2005; Galvan et al., 2006; Eshel et al., 2007). There is general agreement that adolescents tend to show higher VS activation during reward anticipation compared to children and adults (Christakou et al., 2011; Padmanabhan et al., 2011; Galván \& McGlennen, 2013; Barkley-Levenson \& Galván, 2014). ${ }^{2}$ Increased activation in the VS in response to immediate monetary rewards during adolescence has been associated with engagement in harmful behaviors, including alcohol abuse, smoking, dysfunctional eating, and drug addiction (Loxton \& Dawe, 2001; Kambouropoulos \& Staiger, 2004; O’Connor \& Colder, 2005; Crews et al., 2007; Goudriaan et al., 2008; Peeters et al., 2017). However, studies also suggest that heightened sensitivity to reward in the VS can enhance development by increasing motivation in positive types of learning (Cohen et al., 2010; van den Bos et al., 2012; Davidow et al., 2016; Gerraty et al., 2017).

1 It has been theorized that changes in brain function and corresponding behavior should not be attributed to developmental changes in specific brain regions; rather, systemic changes throughout the brain influence responses within brain regions and the strengths of the connections between them (Crone \& Dahl, 2012).

2 Note that some studies have found dampened VS responses (Bjork et al., 2004; Bjork et al., 2010; Lamm et al., 2014) or similar responses to rewards in adolescents compared to adults (Krain et al., 2006; van Leijenhorst et al., 2006; Teslovich et al., 2014). Divergent findings are likely the result of the heterogeneity of task paradigms used to study the developing brain (for a review, see Richards et al., 2013). 
More recent research that examines reward sensitivity in contexts other than direct monetary rewards suggests that heightened sensitivity to other types of reward (e.g., donation to the participant's family, redeemable game points) predicts declines in depressive symptoms (Telzer et al., 2014) and improved cognitive performance (Geier \& Luna, 2012). Increased reward sensitivity is likely part of the normative, adaptive developmental process that motivates adolescents to engage in behaviors that support the successful transition to adulthood (Spielberg et al., 2014). In sum, heightened sensitivity in the value system signals both positive and negative outcomes, depending on the context and the type of reward and social context.

In addition to a general heightened sensitivity during adolescents, value computation that integrates choices with multiple attributes is thought to also develop during adolescence. Decisions are often made among options that differ along multiple attributes, requiring computation and integration of distinct value signals (Montague \& Berns, 2002; Rangel et al., 2008). Neuroimaging studies have consistently shown that integrated subjective value signals are encoded in the VMPFC (Hare et al., 2009; Bartra et al., 2013). A number of studies have examined how neural computation of value differs across development in the context of food choice (Lim et al., 2016; van Meer et al., 2017). For instance, a recent study compared neural responses to healthy and unhealthy food stimuli between early adolescents (10-12 years old) and adults (32-52 years old) (van Meer et al., 2017). The results of this study suggest that although both early adolescent and adult VMPFC activations were modulated by the tastiness of the food, only adult VMPFC activation was modulated by healthiness. These results indicate that when making decisions that include multiple attributes, adolescents may show distinct neural value computation compared to adults, influencing downstream decision-making.

Reward and value processing in the VMPFC and VS are not the exclusive determinants of adolescent decision-making. Other brain systems, including those involved in cognitive control and social cognition, are believed to be involved in valuation and decision-making processes (Steinberg, 2004; Geier \& Luna, 2009). Cognitive control refers to the ability to flexibly and adaptively coordinate behavior to achieve internal goals and includes a variety of interacting components, such as working memory, attention allocation and response inhibition (Badre, 2011; Luna et al., 2015). Adolescents' heightened reward sensitivity may be partially explained by the relatively slower maturation of cognitive control systems compared to the VS (Casey et al., 2008; Sturman \& Moghaddam, 2011; Pfeifer \& Allen, 2012). A study comparing children, adolescents and young adults' neural responses to risky decision-making found that, compared to adolescents, adults relied more on the prefrontal 
cortex (PFC), a set of brain regions implicated in executive and deliberative processing, when making risky choices (Eshel et al., 2007). Other research that explored the functional connectivity between brain regions showed decreased connectivity between the VS and VMPFC and increased connectivity between the lateral PFC and VS across adolescence (van Duijvenvoorde et al., 2016), suggesting that the adolescent valuation system is increasingly influenced by the lateral PFC, a brain region thought to index cognitive control (Wagner et al., 2001). Hence, the changes in connectivity between the cognitive control and motivational systems and shifts in activation of these systems when processing rewards contribute to developmental changes in behavior.

Adolescence is marked by increased neural sensitivity to social cues (Crone $\&$ Dahl, 2012), resulting in a more pronounced influence of social factors on behavior. This neural development is considered adaptive, as adolescents begin to spend more time with peers, navigate social relationships without parental support and develop social skills that are important for adulthood (Blakemore \& Mills, 2014). Subjective valuation during adolescence shows heightened sensitivity to social influence compared to children and adults. In one study that examined the influence of peers on valuation processing in adolescents (ages 14-19) and adults (ages 25-35), participants were instructed to perform a reward processing task both alone and with same-sex, same-aged peers (Smith et al., 2015). The reward processing task required participants to make a series of guesses about the number on a card, and each correct guess was followed by a reward cue. Compared to adults, adolescent VS neural activity increased more with peer presence, suggesting that adolescent VS activity to reward is more sensitive to peer observation. In addition, studies of reward processing that incorporate more explicit social information, such as social norms, suggest that social information can influence adolescent behavior by modulating neural signals related to value processing (Sherman et al., 2016; Welborn et al., 2016). Behaviorally, adolescents are more likely to conform to the perceived social norms of their peers compared to adults (for a review, see Chein, 2015). Neuroimaging studies in this area demonstrate that peer contexts affect adolescent decision-making by influencing brain regions associated with social and value processing (Chein et al., 2011; Peake et al., 2013; Cascio et al., 2015). In sum, the adolescent value system is more sensitive to both implicit social cues and explicit normative social information compared to adults.

Taken together, findings from these studies suggest that the neural valuation system undergoes significant functional specialization and may result in increased reward sensitivity during this developmental period. Moreover, the adolescent valuation system is dependent on social context and is increasingly 
influenced by the still maturing cognitive control system. It is important to acknowledge that brain maturation does not occur independent of other factors. Instead, learning and experiences during adolescence shape developing neural networks in important ways (Takesian \& Hensch, 2013). Nevertheless, these neurodevelopmental changes may serve as neural underpinnings for the observed behavioral traits in adolescents, including the motivation to seek novel, exciting and high-sensation experiences. Considering adolescence as an important time period of exploration and learning, these neurodevelopmental changes are considered critical to supporting healthy psychosocial development during adolescence. For example, this neural development may be necessary in supporting adolescents to step outside their comfort zone, learn a new sport or entertain alternative belief systems. In the next section, we discuss the implications of recent communication and developmental neuroimaging studies on adolescent media health campaigns and consider how neuroimaging tools may help improve adolescent health persuasion through media platforms.

\section{Implications for adolescent media health promotion}

Recent advances in social and communication neuroscience offer insights about the neural mechanisms that underlie adult health communication. Evidence suggests that neural activity in the brain's valuation system plays an important role in health persuasion outcomes, including behavior change and message sharing (Cooper et al., 2015; Riddle et al., 2016; Baek et al., 2017; Cooper et al., 2017; Scholz et al., 2017; Vezich et al., 2017). Considering findings that highlight the changes in the valuation system over the course of adolescence, the extent to which insights from the adult persuasion neuroscience literature can be applied to adolescents is an open question. Combined findings from persuasion and developmental neuroscience underscore at least two possible ways that neuroscience can inform the promotion of health behavior in adolescents.

\section{Explaining additional variance in adolescent health behavior}

A direct application of neuroimaging methods to adolescent health promotion is that they could improve our ability to predict attitudes and behavior based on neural responses to health messaging. First, studies in adult populations demonstrate that neural signals during message exposure can predict individual- and population-level behavior change and often provide information that is different from complementary methods such as questionnaires, explaining up to an additional quarter of the variance in health-relevant outcomes 
compared to survey measures alone (Falk et al., 2010; Falk et al., 2011; Riddle et al., 2016; for a review, see Falk \& Scholz, 2018). Additionally, value-related neural activity in adults during message exposure is associated with the intention to share at the individual level as well as population-level message virality.

Some studies have begun to use neural activation to predict relevant outcomes of health campaign messages in adolescents. One study directly compared adolescents' (15-19 years old) neural activation to strong (convincing) versus weak PSAs as rated by a group of independent raters. The findings demonstrated that stronger PSAs elicited significantly more activation in the lateral PFC, a brain region implicated in executive processing (Ramsay et al., 2013). The authors interpreted this result as indicative of a cognitive control process that translates the persuasive anti-drug message into internal goals. Another study examined adolescents' neural responses to anti-smoking PSAs from a national smoking prevention campaign and found that increased brain activation in the VMPFC (a region of the brain involved in valuation) during message exposure was associated with the degree to which participants talked about the central theme of the message when they verbally shared their opinions on the ads (Pei et al., 2018). These studies provide initial support that the brain imaging paradigms used to study adult health persuasion can be applied to adolescents, even though the neural mechanisms that underlie persuasion may be different. Notably, neither study directly linked adolescent neural activity during health message exposure with behavior change. Future neuroimaging studies that include individual-level and population-level communication outcomes in adolescents will help us better understand the neuropsychological processes that underlie health persuasion and how they change with development and inform more effective campaign design.

Neuroimaging tools can also forecast message sharing, which is also integral for population-level health campaign success. In adults, neural activation in brain regions involved in self-relevance and social cognition contribute to the value of sharing a message (Baek et al., 2017; Scholz et al., 2017). Future work in adolescent participants can help determine the extent to which neural signals in the same or additional regions can predict message sharing. These insights can inform the development of messages that are both effective and shareworthy, thus maximizing the reach and overall success of a campaign.

More broadly, the ability to predict behavior based on neural responses to messages in adolescents depends critically on the understanding of the cognitive mechanisms leading to effective persuasion (Suleiman \& Dahl, 2017). For instance, knowledge about the cognitive mechanisms that lead to successful persuasion or message retransmission can illuminate key brain regions involved, which may differ among different types of campaigns (e.g., prevention-focused campaigns or campaigns aimed at changing a certain behavior) 
and in different adolescent populations (e.g., high sensation-seeking adolescents). In turn, studies that utilize neural signals to predict relevant health campaign outcomes can potentially inform our understanding and theorization of the mechanisms underlying persuasion in adolescents, which we discuss in more detail in the following section.

\section{Understanding the underlying processes}

In addition to complementing current tools for predicting campaign message effectiveness, neuroscience studies on adolescent development and persuasion can reveal common and distinct underlying processes that may be involved in adolescent persuasion and highlight the relationships among these processes. For example, recent adult neuroimaging evidence brings together several theories of persuasion under a more general framework of value-based decisionmaking (for a review, see Falk \& Scholz, 2018). Additional insights into the underlying processes of adolescent persuasion could also inform media campaigns aimed at promoting adolescent health and well-being. Here, we discuss two sets of findings that may be relevant to health promotion: redirecting reward-seeking adolescents to engage in rewarding, pro-health behavior; and utilizing the power of social media.

Adolescents generally show heightened neural sensitivity to reward (Galvan et al., 2006; Somerville \& Casey, 2010; van Duijvenvoorde et al., 2014). Although individuals with heightened reward sensitivity demonstrate increased vulnerability to engaging in substance use, dysfunctional eating and risky driving (Loxton \& Dawe, 2001; O'Connor \& Colder, 2005; Brenhouse \& Andersen, 2011; Spear, 2011; Galván \& McGlennen, 2013; Scott-Parker et al., 2013), higher reward sensitivity can also lead to developmentally adaptive outcomes such as better inhibitory control (Padmanabhan et al., 2011), decreases in risky, delinquent behaviors (e.g., drinking alcohol, stealing, skipping school, using drugs) (Telzer et al., 2013) and declines in depressive symptoms (Telzer et al., 2014) in certain contexts. Evidence for both the adaptive and maladaptive consequences of heightened reward sensitivity suggests that, instead of a vulnerability to risky decisions that could harm adolescents' health and well-being, adolescents' heightened reward sensitivity should be considered a neurobiological marker for domain-general, approach-related behaviors that can also be channeled to promote positive outcomes (Telzer, 2016).

With this perspective in mind, an important open question is how to design health media campaigns that optimally promote the positive, pro-social tendencies for risk-taking while minimizing harmful outcomes. In other words, how can health professionals channel adolescents' motivations to engage in 
high-stakes risk-taking and direct them toward behaviors that result in increased health and well-being? One possible approach is to emphasize values that are salient for adolescents, such as values relevant to social status, peer relationships, social acceptance and romantic interests in promoting positive rather than avoiding negative behaviors (Suleiman \& Dahl, 2017). A recent study showed that, compared to traditional education messages, health messages that tap into adolescents' need for autonomy and desire for social respect were more successful at influencing adolescent healthy eating behaviors (Bryan et al., 2016). Similarly, in addition to the negative consequences of smoking, a campaign aimed at preventing adolescents from smoking could also leverage adolescence as a period of flexibility, exploration and learning to highlight pro-health values associated with smoking abstinence, such as an increased capacity for physical activity and improved social relationships. Given the social, psychological and behavioral complexities of health behavior and adolescent development, however, the corresponding health promotion strategies are likely to be equally nuanced and require direct input from adolescents.

Another direction in which insights from developmental and persuasion neuroscience studies could be helpful is the use of new media to disseminate and promote health messages. Social media is ubiquitous with regard to adolescent social networking and content sharing. Health campaigns increasingly use social media to disseminate health campaign messages in an attempt to influence attitudes and behaviors. Social media afford important functions such as content sharing and user commenting, supporting a greater extent of user engagement and participation compared to traditional mass media (e.g., TV, radio, and newspapers) (Neiger et al., 2012; Thackeray et al., 2008; Moorhead et al., 2013). Sharing of campaign content on social media may create opportunities for exposure to individuals who would otherwise be unexposed, which may in turn support the diffusion of messages to a wider audience and improve campaign effectiveness (Hornik \& Yanovitzky, 2003). Furthermore, liking, sharing and commenting behaviors by peers may provide social cues and social information in addition to the original health message. In a recent neuroimaging study, adolescents aged 13-18 years old were shown images that simulated photographs shared on Instagram, a social media platform, while in an fMRI scanner (Sherman et al., 2016). The results demonstrated that viewing pictures with many (compared with few) likes was associated with greater neural activity in brain regions implicated in reward processing, social cognition and attention, suggesting that the conferral of social approval from peers may increase the value of mediated messages and subsequently enhance message effects. Given adolescents' heightened sensitivity to social evaluation (Crone \& Dahl, 2012; Albert et al., 2013), 
the social information (e.g., the sharing of a health message by one's friend) may facilitate persuasion among those who would not otherwise be persuaded by a health message. Finally, social media offer the opportunity for audience engagement, both with health professionals and with peers (for a review, see Heldman et al., 2013). Elements of peer engagement and social support are particularly relevant for adolescent health promotion through social media; however, these same features can also backfire if comments and interpersonal communications about the campaign are negative (Hendriks et al., 2014). As such, it is important to understand the developmental trajectories and contextual influences that may determine the positive or negative direction of social influence. Systematic investigations are needed into whether and how health campaign professionals can manage a social media presence that promotes positive social feedback and community engagement within the affordances of social media sites. This action is crucial, as negative social feedback can not only undermine campaign message effectiveness, but also lead to boomerang effects (unintended consequences of attempts to persuade that result in opposite outcomes) (Southwell \& Yzer, 2009).

\section{Conclusion: bridging from evidence to policy}

Adolescence is a critical developmental period during which there is considerable opportunity for establishing life-long health-promoting behaviors. Conversely, there are great risks of initiating poor health behaviors that may be carried into adulthood. These point to the importance of gaining a better understanding of the brain's valuation system in adolescent health persuasion.

Given evidence that the brain undergoes significant development during adolescence, it is important to explore the ways in which development affects valuation. The understanding that the adolescent brain is undergoing functional reorganization and is more sensitive to social cues suggests that specific types of messages may be most effective during specific developmental windows. Integrating insights about neurodevelopmental trajectories with social and contextual factors that influence brain development (e.g., family support, social relationships, prior experiences) can further enhance the precision of these messages. Thus, investigations of the neurodevelopmental changes that occur during adolescence provide insights into the mechanisms that underlie successful health persuasion, as well as the potential to more accurately forecast which messages will work for which groups of adolescents.

As reviewed above, adolescents' heightened sensitivity to rewards should be considered as a motivational force that facilitates learning and exploring, which can both increase vulnerabilities for unhealthy behaviors and increase opportunities for positive behaviors. Media campaign messages that effectively 
promote pro-health, pro-social channels of reward-seeking may prove more effective than those that focus solely on decreasing unhealthy and risky behaviors. Testing this hypothesis holds great promise for improving adolescent health trajectories. Finally, the content-sharing and commenting features of social media platforms provide opportunities for peer and community influence that could be particularly effective when communicating with adolescents. Many efforts are already being made to integrate social media into health promotion campaigns, and communication neuroscience offers strategies to streamline these efforts and ensure that the most effective messages reach the right audiences. Collectively, bringing together the fields of adolescent developmental science and communication neuroscience has the potential to significantly improve the health trajectories of young people.

\section{Acknowledgements}

We thank Robert Hornik, Nicole Cooper and Ron Dahl for their very valuable comments and discussions. Preparation of this manuscript was supported by the NIH New Innovator Award 1DP2DA03515601 (PI Falk), the Defense Advanced Research Projects Agency (DARPA) Grant D14AP00048 (PI Falk), the Army Research Laboratory under Cooperative Agreement Number W911NF-10-2-0022 (PI Falk) and HopeLab. The funding agencies played no role in the development of this manuscript. The views expressed in this manuscript are those of the authors and should not be interpreted as representing the views or policies of the funding agencies.

\section{References}

Albarracin, D. and S. Shavitt (2018), 'Attitudes and Attitude Change'. Annual review of psychology, 69, 299-327.

Albert, D., J. Chein and L. Steinberg (2013), 'Peer Influences on Adolescent Decision Making', Current directions in psychological science, 22(2): 114-120.

Atkin, C. K. and V. S. Freimuth (2001), 'Formative evaluation research in campaign design', Public communication campaigns, 3, 125-145.

Aubrey, J. S., E. Behm-Morawitz and K. Kim (2014), 'Understanding the effects of MTV's 16 and Pregnant on adolescent girls' beliefs, attitudes, and behavioral intentions toward teen pregnancy', Journal of health communication, 19(10): 1145-1160.

Badre, D. (2011), 'Defining an ontology of cognitive control requires attention to component interactions', Topics in cognitive science, 3(2): 217-221.

Baek, E. C., C. Scholz, M. B. O’Donnell and E. B. Falk (2017), 'The Value of Sharing Information: A Neural Account of Information Transmission', Psychological science, 28(7): 851-861.

Barkley-Levenson, E. and A. Galván (2014), 'Neural representation of expected value in the adolescent brain', Proceedings of the National Academy of Sciences of the United States of America, 111(4): 1646-1651.

Bartra, O., J. T. McGuire and J. W. Kable (2013), 'The valuation system: a coordinate-based metaanalysis of BOLD fMRI experiments examining neural correlates of subjective value', NeuroImage, 76, 412-427. 
Berger, J. (2014), 'Word of mouth and interpersonal communication: A review and directions for future research', Journal of consumer psychology: the official journal of the Society for Consumer Psychology, 24(4): 586-607.

Berkman, E. T. and E. B. Falk (2013), 'Beyond Brain Mapping: Using Neural Measures to Predict Real-World Outcomes', Current directions in psychological science, 22(1): 45-50.

Berns, G. S. and S. E. Moore (2012), 'A neural predictor of cultural popularity', Journal of consumer psychology: the official journal of the Society for Consumer Psychology, 22(1): 154-160.

Bjork, J. M., A. R. Smith, G. Chen and D. W. Hommer (2010), 'Adolescents, adults and rewards: comparing motivational neurocircuitry recruitment using fMRI', PLoS One, 5(7), e11440.

Bjork, J. M., B. Knutson, G. W. Fong, D. M. Caggiano, S. M. Bennett and D. W. Hommer (2004), 'Incentive-Elicited Brain Activation in Adolescents: Similarities and Differences from Young Adults', The Journal of neuroscience: the official journal of the Society for Neuroscience, 24 (8): 1793-1802.

Blakemore, S. J. and K. L. Mills (2014), 'Is Adolescence a Sensitive Period for Sociocultural Processing?', Annual review of psychology, 65(1): 187-207.

Boksem, M. A. S. and A. Smidts (2014), 'Brain Responses to Movie Trailers Predict Individual Preferences for Movies and Their Population-Wide Commercial Success', JMR, Journal of marketing research, 52(4): 482-492.

Braams, B. R., A. C. K. van Duijvenvoorde, J. S. Peper and E. A. Crone (2015), 'Longitudinal changes in adolescent risk-taking: a comprehensive study of neural responses to rewards, pubertal development, and risk-taking behavior', The Journal of neuroscience: the official journal of the Society for Neuroscience, 35(18): 7226-7238.

Brenhouse, H. C. and S. L. Andersen (2011), 'Developmental trajectories during adolescence in males and females: a cross-species understanding of underlying brain changes', Neuroscience and biobehavioral reviews, 35(8): 1687-1703.

Brennan, E., S. J. Durkin, M. A. Wakefield and Y. Kashima (2016), 'Talking About Antismoking Campaigns: What Do Smokers Talk About, and How Does Talk Influence Campaign Effectiveness?' Journal of health communication, 21(1): 33-45.

Brennan, E., A. Momjian, M. Jeong, D. Naugle, S. Parvanta and R. Hornik (2012), Mass media campaigns to reduce smoking among youth and young adults: Documenting potential campaign targets and reviewing the evidence from previous campaigns (CECCR Working Paper Series). Philadelphia, PA: Penn's Center of Excellence in Cancer Communication Research, Annenberg School for Communication, University of Pennsylvania.

Bryan, C. J., D. S. Yeager, C. P. Hinojosa, A. Chabot, H. Bergen, M. Kawamura and F. Steubing (2016), 'Harnessing adolescent values to motivate healthier eating', Proceedings of the National Academy of Sciences of the United States of America, 113(39): 10830-10835.

Cacioppo, J. T., S. Cacioppo and R. E. Petty (2016), 'The neuroscience of persuasion: A review with an emphasis on issues and opportunities', Social neuroscience, 0(0): 1-44.

Cappella, J. N. (2006), 'Integrating Message Effects and Behavior Change Theories: Organizing Comments and Unanswered Questions', The Journal of communication, 56, S265-S279.

Cascio, C. N., M. B. O’Donnell, J. Bayer, F. J. Tinney and E. B. Falk (2015), 'Neural Correlates of Susceptibility to Group Opinions in Online Word-of-Mouth Recommendations', JMR, Journal of marketing research, 52(4): 559-575.

Casey, B. J., R. M. Jones and T. A. Hare (2008), 'The Adolescent Brain', Annals of the New York Academy of Sciences, 1124, 111-126.

Chein, J. (2015), 'Peers and Adolescent Risk Taking', in: Emerging Trends in the Social and Behavioral Sciences, John Wiley \& Sons, Inc.

Chein, J., D. Albert, L. O’Brien, K. Uckert and L. Steinberg (2011), 'Peers increase adolescent risk taking by enhancing activity in the brain's reward circuitry', Developmental science, 14(2): F1-F10. 
Christakou, A., M. Brammer and K. Rubia (2011), 'Maturation of limbic corticostriatal activation and connectivity associated with developmental changes in temporal discounting', NeuroImage, 54(2): 1344-1354.

Chua, H. F., S. S. Ho, A. J. Jasinska, T. A. Polk, R. C. Welsh, I. Liberzon and V. J. Strecher (2011), 'Self-related neural response to tailored smoking-cessation messages predicts quitting', Nature neuroscience, 14(4): 426-427.

Cialdini, R. B. and N. J. Goldstein (2004), 'Social influence: compliance and conformity', Annual review of psychology, 55, 591-621.

Cohen, J. R., R. F. Asarnow, F. W. Sabb, R. M. Bilder, S. Y. Bookheimer, B. J. Knowlton and R. A. Poldrack (2010), 'A unique adolescent response to reward prediction errors', Nature neuroscience, 13(6): 669-671.

Cooper, N., D. S. Bassett and E. B. Falk (2017), 'Coherent activity between brain regions that code for value is linked to the malleability of human behavior', Scientific reports, 7: 43250.

Cooper, N., S. Tompson, M. Brook O'Donnell and B. F. Emily (2015), 'Brain Activity in Self- and Value-Related Regions in Response to Online Antismoking Messages Predicts Behavior Change', Journal of Media Psychology, 27(3): 93-109.

Crews, F., J. He and C. Hodge (2007), 'Adolescent cortical development: A critical period of vulnerability for addiction', Pharmacology, biochemistry, and behavior, 86(2): 189-199.

Crone, E. A. and R. E. Dahl (2012), 'Understanding adolescence as a period of social-affective engagement and goal flexibility', Nature reviews. Neuroscience, 13(9): 636-650.

Dahl, R. E. (2004), 'Adolescent Brain Development: A Period of Vulnerabilities and Opportunities', Keynote Address. Annals of the New York Academy of Sciences, 1021(1): 1-22.

Davidow, J. Y., K. Foerde, A. Galván and D. Shohamy (2016), 'An Upside to Reward Sensitivity: The Hippocampus Supports Enhanced Reinforcement Learning in Adolescence', Neuron, 92(1): 93-99.

Dijksterhuis, A. (2004), 'Think different: the merits of unconscious thought in preference development and decision making', Journal of personality and social psychology, 87(5): 586-598.

Ellis, B. J., M. Del Giudice, T. J. Dishion, A. J. Figueredo, P. Gray, V. Griskevicius, P. H. Hawley, W. Jake Jacobs, J. James, A. A. Volk and D. S. Wilson (2012), 'The Evolutionary Basis of Risky Adolescent Behavior: Implications for Science, Policy, and Practice', Developmental psychology, 48(3): 598-623.

Ernst, M., E. E. Nelson, S. Jazbec, E. B. McClure, C. S. Monk, E. Leibenluft, J. Blair and D. S. Pine (2005), 'Amygdala and nucleus accumbens in responses to receipt and omission of gains in adults and adolescents', NeuroImage, 25(4): 1279-1291.

Eshel, N., E. E. Nelson, R. J. Blair, D. S. Pine and M. Ernst (2007), 'Neural substrates of choice selection in adults and adolescents: Development of the ventrolateral prefrontal and anterior cingulate cortices', Neuropsychologia, 45(6): 1270-1279.

Falk, E. B., E. T. Berkman and M. D. Lieberman (2012), 'From Neural Responses to Population Behavior Neural Focus Group Predicts Population-Level Media Effects', Psychological science, 0956797611434964.

Falk, E. B., E. T. Berkman, T. Mann, B. Harrison and M. D. Lieberman (2010), 'Predicting Persuasion-Induced Behavior Change from the Brain', The Journal of neuroscience: the official journal of the Society for Neuroscience, 30(25): 8421-8424.

Falk, E. B., E. T. Berkman, D. Whalen and M. D. Lieberman (2011), 'Neural activity during health messaging predicts reductions in smoking above and beyond self-report', Health Psychology, 30(2): $177-185$.

Falk, E. B., C. N. Cascio, M. B. O’Donnell, J. Carp, F. J. Tinney, C. R. Bingham, J. T. Shope, M. C. Ouimet, A. K. Pradhan and B. G. Simons-Morton (2014), 'Neural responses to exclusion 
predict susceptibility to social influence', The Journal of adolescent health: official publication of the Society for Adolescent Medicine, 54(5 0): S22-S31.

Falk, E. B., M. B. O’Donnell, C. N. Cascio, F. Tinney, Y. Kang, M. D. Lieberman, S. E. Taylor, L. An, K. Resnicow and V. J. Strecher (2015), 'Self-affirmation alters the brain's response to health messages and subsequent behavior change', Proceedings of the National Academy of Sciences of the United States of America, 112(7): 1977-1982.

Falk, E. B., M. B. O'Donnell, S. Tompson, R. Gonzalez, S. Dal Cin, V. Strecher, K. M. Cummings and L. An (2016), 'Functional brain imaging predicts public health campaign success', Social cognitive and affective neuroscience, 11(2): 204-214.

Falk, E. and C. Scholz (2018), 'Persuasion, Influence, and Value: Perspectives from Communication and Social Neuroscience', Annual review of psychology, 69(1): null.

Farrelly, M. C., K. C. Davis, M. L. Haviland, P. Messeri and C. G. Healton (2005), 'Evidence of a dose-response relationship between 'truth' antismoking ads and youth smoking prevalence', American journal of public health, 95(3): 425-431.

Farrelly, M. C., J. Niederdeppe and J. Yarsevich (2003), 'Youth tobacco prevention mass media campaigns: past, present, and future directions', Tobacco control, 12(suppl 1), i35-i47.

Fazio, R. H. and M. A. Olson (2003), 'Implicit Measures in Social Cognition Research: Their Meaning and Use', Annual review of psychology, 54(1): 297-327.

Fishbein, M. and I. Ajzen (2011), Predicting and Changing Behavior: The Reasoned Action Approach, Taylor \& Francis.

Galvan, A., T. A. Hare, C. E. Parra, J. Penn, H. Voss, G. Glover and B. J. Casey (2006), 'Earlier development of the accumbens relative to orbitofrontal cortex might underlie risk-taking behavior in adolescents', The Journal of Neuroscience, 26(25): 6885-6892.

Galván, A. and K. M. McGlennen (2013), 'Enhanced striatal sensitivity to aversive reinforcement in adolescents versus adults', Journal of cognitive neuroscience, 25(2): 284-296.

Geber, S., E. Baumann and C. Klimmt (2017), Where Do Norms Come From? Peer Communication as a Factor in Normative Social Influences on Risk Behavior. Communication research, 0093650217718656.

Geier, C. F. and B. Luna (2012), 'Developmental Effects of Incentives on Response Inhibition', Child development, 83(4): 1262-1274.

Geier, C. and B. Luna (2009), 'The maturation of incentive processing and cognitive control', Pharmacology, biochemistry, and behavior, 93(3): 212-221.

Genevsky, A. and B. Knutson (2015), 'Neural Affective Mechanisms Predict Market-Level Microlending', Psychological science, 26(9): 1411-1422.

Gerraty, R. T., J. Y. Davidow, K. Foerde, A. Galvan, D. S. Bassett and D. Shohamy (2017), 'Dynamic flexibility in striatal-cortical circuits supports reinforcement learning', bioRxiv, 094383.

Goldberg, M. E., M. Fishbein and S. E. Middlestadt (1997), Social marketing: Theoretical and practical perspectives, Lawrence Erlbaum Associates.

Goudriaan, A. E., J. Oosterlaan, E. D. Beurs and W. V. D. Brink (2008), 'The role of self-reported impulsivity and reward sensitivity versus neurocognitive measures of disinhibition and decision-making in the prediction of relapse in pathological gamblers', Psychological medicine, 38(1): 41-50.

Grant, B. F., D. A. Dawson, F. S. Stinson, S. P. Chou, M. C. Dufour and R. P. Pickering (2004), 'The 12-month prevalence and trends in DSM-IV alcohol abuse and dependence: United States, 1991-1992 and 2001-2002', Drug and alcohol dependence, 74(3): 223-234.

Hare, T. A., C. F. Camerer and A. Rangel (2009), 'Self-control in decision-making involves modulation of the vmPFC valuation system', Science, 324(5927): 646-648. 
Heldman, A. B., J. Schindelar and J. B. Weaver (2013), 'Social Media Engagement and Public Health Communication: Implications for Public Health Organizations Being Truly “Social”, Public health reviews, 35(1): 13 .

Hendriks, H., B. van den Putte, G.-J. de Bruijn and C. H. de Vreese (2014), 'Predicting health: the interplay between interpersonal communication and health campaigns', Journal of health communication, 19(5): 625-636.

Hornik, R. (2002), Public Health Communication: Evidence for Behavior Change, Routledge.

Hornik, R. and I. Yanovitzky (2003), Using Theory to Design Evaluations of Communication Campaigns: The Case of the National Youth Anti-Drug Media Campaign. Communication theory: CT: a journal of the International Communication Association., 204-224.

Jessor, R. (1984), 'Adolescent development and heavioral health', in Behavioral health: A handbook of health enhancement and disease prevention, New York: Wiley, 69-90.

Jordan, A. B. and D. Romer (2014), Media and the Well-Being of Children and Adolescents, Oxford University Press.

Kambouropoulos, N. and P. K. Staiger (2004), 'Reactivity to alcohol-related cues: relationship among cue type, motivational processes, and personality', Psychology of Addictive Behaviors, 18(3): $275-283$.

Katz, E. (1957), 'The Two-Step Flow of Communication: An Up-To-Date Report on an Hypothesis', Public opinion quarterly, 21(1): 61-78.

Katz, E. and P. F. Lazarsfeld (1955), Personal Influence, the Part Played by People in the Flow of Mass Communications, Glencoe, IL: Free Press.

Kleinert, S. (2007), 'Adolescent health: an opportunity not to be missed', The Lancet, 369(9567): 1057-1058.

Korda, H. and Z. Itani (2013), 'Harnessing social media for health promotion and behavior change', Health promotion practice, 14(1): 15-23.

Krain, A. L., S. Hefton, D. S. Pine, M. Ernst, F. X. Castellanos, R. G. Klein and M. P. Milham (2006), 'An fMRI examination of developmental differences in the neural correlates of uncertainty and decision-making', Journal of Child Psychology and Psychiatry, and Allied Disciplines, 47(10), 1023-1030.

Lamm, C., B. E. Benson, A. E. Guyer, K. Perez-Edgar, N. A. Fox, D. S. Pine and M. Ernst (2014), 'Longitudinal study of striatal activation to reward and loss anticipation from mid-adolescence into late adolescence/early adulthood', Brain and Cognition, 89, 51-60.

Lenhart, A. (2015), Teens, Social Media \& Technology Overview 2015. Pew Research Center: Internet, Science \& Tech. [Online]. [Accessed 24 July 2017]. Available from: http://www. pewinternet.org/2015/04/09/teens-social-media-technology-2015/.

Levy, D. J. and P. W. Glimcher (2012), 'The root of all value: a neural common currency for choice', Current opinion in neurobiology, 22(6): 1027-1038.

Lieberman, M. D. (2010), Social cognitive neuroscience. Handbook of social psychology

Lim, S.-L., J. B. C. Cherry, A. M. Davis, S. N. Balakrishnan, O.-R. Ha, J. M. Bruce and A. S. Bruce (2016), 'The child brain computes and utilizes internalized maternal choices', Nature communications, 7: 11700.

Loxton, N. J. and S. Dawe (2001), 'Alcohol abuse and dysfunctional eating in adolescent girls: the influence of individual differences in sensitivity to reward and punishment', The International journal of eating disorders, 29(4): 455-462.

Luna, B., S. Marek, B. Larsen, B. Tervo-Clemmens and R. Chahal (2015), 'An integrative model of the maturation of cognitive control', Annual review of neuroscience, 38, 151-170.

May, J. C., M. R. Delgado, R. E. Dahl, V. A. Stenger, N. D. Ryan, J. A. Fiez and C. S. Carter (2004), 'Event-related functional magnetic resonance imaging of reward-related brain circuitry in children and adolescents', Biological psychiatry, 55(4): 359-366. 
McCombs, M. E. and D. L. Shaw (1972), 'The agenda-setting function of mass media', Public opinion quarterly, 36(2): 176-187.

Miniño, A. M. (2010), Mortality among teenagers aged 12-19 years: United States, 1999- 2006, Hyattsville, MD: National Center for Health Statistics.

Montague, P. R. and G. S. Berns (2002), 'Neural economics and the biological substrates of valuation', Neuron, 36(2): 265-284.

Moorhead, S. A., D. E. Hazlett, L. Harrison, J. K. Carroll, A. Irwin and C. Hoving (2013), 'A new dimension of health care: systematic review of the uses, benefits, and limitations of social media for health communication', Journal of medical Internet research, 15(4): e85.

Neiger, B. L., R. Thackeray, S. A. Van Wagenen, C. L. Hanson, J. H. West, M. D. Barnes and M. C. Fagen (2012), 'Use of social media in health promotion: purposes, key performance indicators, and evaluation metrics', Health promotion practice, 13(2): 159-164.

Noar, S. M. (2006), 'A 10-Year Retrospective of Research in Health Mass Media Campaigns: Where Do We Go From Here? Journal of health communication, 11(1): 21-42.

O'Connor, R. M. and C. R. Colder (2005), 'Predicting Alcohol Patterns in First-Year College Students Through Motivational Systems and Reasons for Drinking', Psychology of addictive behaviors: journal of the Society of Psychologists in Addictive Behaviors, 19(1): $10-20$.

Owens, M. M., J. MacKillop, J. C. Gray, B. E. Hawkshead, C. M. Murphy and L. H. Sweet (2017), 'Neural correlates of graphic cigarette warning labels predict smoking cessation relapse', Psychiatry Research: Neuroimaging, 262, 63-70.

Padmanabhan, A., C. F. Geier, S. J. Ordaz, T. Teslovich and B. Luna (2011), 'Developmental changes in brain function underlying the influence of reward processing on inhibitory control', Developmental cognitive neuroscience, 1(4): 517-529.

Palmgreen, P., E. P. Lorch, M. T. Stephenson, R. H. Hoyle and L. Donohew (2007), 'Effects of the Office of National Drug Control Policy's Marijuana Initiative Campaign on high-sensationseeking adolescents', American Journal of Public Health, 97(9), 1644-1649.

Paulhus, D. L. (1986), 'Self-Deception and Impression Management in Test Responses', in P. D. A. Angleitner and P. D. J. S. Wiggins, (eds), Personality Assessment via Questionnaires, Springer Berlin Heidelberg, 143-165.

Peake, S. J., T. J. Dishion, E. A. Stormshak, W. E. Moore and J. H. Pfeifer (2013), 'Risk-taking and social exclusion in adolescence: neural mechanisms underlying peer influences on decisionmaking', NeuroImage, 82, 23-34.

Peeters, M., T. Oldehinkel and W. Vollebergh (2017), 'Behavioral Control and Reward Sensitivity in Adolescents' Risk Taking Behavior: A Longitudinal TRAILS Study', Frontiers in psychology, 8.

Pei, R., R. Schmaelzle, E. Kranzler, M. B. O'Donnell and E. B. Falk Under review. Neural activity during anti-smoking message exposure predicts subsequent message elaboration.

Pei, R., R. Schmaelzle, M. B. O’Donnell, E. Kranzler, \& E. B. Falk (2018), 'Adolescents' neural responses to anti-smoking messages and subsequent sharing message engagement', American Journal of Preventive Medicine (in press).

Pfeifer, J. H. and N. B. Allen (2012), 'Arrested development? Reconsidering dual-systems models of brain function in adolescence and disorders', Trends in cognitive sciences, 16(6): 322-329.

Pringle, J., K. Mills, J. McAteer, R. Jepson, E. Hogg, N. Anand and S. J. Blakemore (2016), 'A systematic review of adolescent physiological development and its relationship with healthrelated behaviour: a protocol', Systematic reviews, $5: 3$.

Ramsay, I. S., M. C. Yzer, M. Luciana, K. D. Vohs and A. W. MacDonald (2013), 'Affective and Executive Network Processing Associated with Persuasive Antidrug Messages', Journal of cognitive neuroscience, 25(7): 1136-1147.

Rangel, A., C. Camerer and P. R. Montague (2008), 'A framework for studying the neurobiology of value-based decision making’, Nature reviews. Neuroscience, 9(7): 545-556. 
Richards, J. M., R. C. Plate and M. Ernst (2013), 'A systematic review of fMRI reward paradigms used in studies of adolescents vs. adults: The impact of task design and implications for understanding neurodevelopment', Neuroscience \& Biobehavioral Reviews, 37(5), 976-991.

Riddle, P. J., R. D. Newman-Norlund, J. Baer and J. F. Thrasher (2016), 'Neural response to pictorial health warning labels can predict smoking behavioral change', Social cognitive and affective neuroscience, 11(11): 1802-1811.

Rideout, V. (2016), 'Measuring time spent with media: the Common Sense census of media use by US 8- to 18-year-olds', Journal of Children and Media, 10(1): 138-144.

Rus, H. M. and L. D. Cameron (2016), 'Health Communication in Social Media: Message Features Predicting User Engagement on Diabetes-Related Facebook Pages', Annals of behavioral medicine: a publication of the Society of Behavioral Medicine, 50(5): 678-689.

Scholz, C., E. C. Baek, M. B. O'Donnell, H. S. Kim, J. N. Cappella and E. B. Falk (2017), 'A neural model of valuation and information virality', Proceedings of the National Academy of Sciences of the United States of America, 114(11): 2881-2886.

Scott-Parker, B., B. Watson, M. J. King and M. K. Hyde (2013), 'A further exploration of sensation seeking propensity, reward sensitivity, depression, anxiety, and the risky behaviour of young novice drivers in a structural equation model', Accident; analysis and prevention, 50, 465471.

Sherman, L. E., A. A. Payton, L. M. Hernandez, P. M. Greenfield and M. Dapretto (2016), The Power of the Like in Adolescence: Effects of Peer Influence on Neural and Behavioral Responses to Social Media. Psychological science. [Online]. Available from: http://dx.doi.org/10.1177/ 0956797616645673.

Simons-Morton, B., N. Lerner and J. Singer (2005), 'The observed effects of teenage passengers on the risky driving behavior of teenage drivers', Accident; Analysis and Prevention, 37(6): 973-982.

Smith, A. R., L. Steinberg, N. Strang and J. Chein (2015), 'Age Differences inthe impact of peers on adolescents' and adults' neural response to reward', Developmental cognitive neuroscience, (11), 75-82.

Somerville, L. H. and B. J. Casey (2010), 'Developmental neurobiology of cognitive control and motivational systems', Current Opinion in Neurobiology, 20(2), 236-241.

Southwell, B. G. and M. C. Yzer (2009), 'When (and Why) Interpersonal Talk Matters for Campaigns', Communication theory: CT: a journal of the International Communication Association, 19(1), 1-8.

Spear, L. P. (2011), 'Rewards, aversions and affect in adolescence: Emerging convergences across laboratory animal and human data', Developmental cognitive neuroscience, 1(4): 390-403.

Spielberg, J. M., T. M. Olino, E. E. Forbes and R. E. Dahl (2014), 'Exciting fear in adolescence: does pubertal development alter threat processing? Developmental cognitive neuroscience, 8, 86-95.

Steinberg, L. (2014), Age of Opportunity: Lessons from the New Science of Adolescence, Houghton Mifflin Harcourt.

Steinberg, L. (2004), 'Risk taking in adolescence: what changes, and why? Annals of the New York Academy of Sciences, 1021, 51-58.

Steinberg, L. and A. S. Morris (2001), 'Adolescent development', Annual review of psychology, 52, 83-110.

Sturman, D. A. and B. Moghaddam (2011), 'Reduced neuronal inhibition and coordination of adolescent prefrontal cortex during motivated behavior', The Journal of neuroscience: the official journal of the Society for Neuroscience, 31(4): 1471-1478.

Suleiman, A. B. and R. E. Dahl (2017), 'Leveraging Neuroscience to Inform Adolescent Health: The Need for an Innovative Transdisciplinary Developmental Science of Adolescence', Journal of Adolescent Health Care: official publication of the Society for Adolescent Medicine, 60(3): 240-248. 
Takesian, A. E. and T. K. Hensch (2013), 'Balancing Plasticity/Stability Across Brain Development', Progress in brain research, 207, 3-34.

Telzer, E. H. (2016), 'Dopaminergic reward sensitivity can promote adolescent health: A new perspective on the mechanism of ventral striatum activation', Developmental cognitive neuroscience, 17, 57-67.

Telzer, E. H., A. J. Fuligni, M. D. Lieberman and A. Galván (2014), 'Neural sensitivity to eudaimonic and hedonic rewards differentially predict adolescent depressive symptoms over time', Proceedings of the National Academy of Sciences of the United States of America, 111(18): 6600-6605.

Telzer, E. H., A. J. Fuligni, M. D. Lieberman and A. Galván (2013), 'The effects of poor quality sleep on brain function and risk taking in adolescence', NeuroImage, 71, 275-283.

Teslovich, T., M. Mulder, N. T. Franklin, E. J. Ruberry, A. Millner, L. H. Somerville, ... B. J. Casey (2014), 'Adolescents let sufficient evidence accumulate before making a decision when large incentives are at stake', Developmental Science, 17(1), 59-70.

Thackeray, R., B. L. Neiger, C. L. Hanson and J. F. McKenzie (2008), 'Enhancing promotional strategies within social marketing programs: use of Web 2.0 social media', Health promotion practice, 9(4): 338-343.

van den Bos, W., M. X. Cohen, T. Kahnt and E. A. Crone (2012), 'Striatum-Medial Prefrontal Cortex Connectivity Predicts Developmental Changes in Reinforcement Learning', Cerebral cortex, . 22(6): 1247-1255.

van Duijvenvoorde, A. C. K., Z. A. Op de Macks, S. Overgaauw, B. Gunther Moor, R. E. Dahl and E. A. Crone (2014), 'A cross-sectional and longitudinal analysis of reward-related brain activation: Effects of age, pubertal stage, and reward sensitivity', Brain and Cognition, 89, 3-14.

van Duijvenvoorde, A. C. K., S. Peters, B. R. Braams and E. A. Crone (2016), 'What motivates adolescents? Neural responses to rewards and their influence on adolescents' risk taking, learning, and cognitive control', Neuroscience and biobehavioral reviews, 70, 135-147.

van Leijenhorst, L., E. A. Crone and S. A. Bunge (2006), 'Neural correlates of developmental differences in risk estimation and feedback processing', Neuropsychologia, 44(11), 2158-2170.

van Meer, F., L. N. van der Laan, M. A. Viergever, R. A. H. Adan, P. A. M. Smeets and I.Family Consortium (2017), 'Considering healthiness promotes healthier choices but modulates medial prefrontal cortex differently in children compared with adults', NeuroImage, 159, $325-333$.

Vezich, I. S., P. L. Katzman, D. L. Ames, E. B. Falk and M. D. Lieberman (2017), 'Modulating the neural bases of persuasion: why/how, gain/loss, and users/non-users', Social cognitive and affective neuroscience, 12(2): 283-297.

Wagner, A. D., A. Maril, R. A. Bjork and D. L. Schacter (2001), 'Prefrontal Contributions to Executive Control: fMRI Evidence for Functional Distinctions within Lateral Prefrontal Cortex', NeuroImage, 14(6): 1337-1347.

Wakefield, M. A., B. Loken and R. C. Hornik (2010), 'Use of mass media campaigns to change health behaviour', The Lancet, 376(9748): 1261-1271.

Wakefield, M., B. Flay, M. Nichter and G. Giovino (2003), 'Effects of Anti-Smoking Advertising on Youth Smoking: A Review', Journal of health communication, 8(3): 229-247.

Wang, A.-L., S. B. Lowen, D. Romer, M. Giorno and D. D. Langleben (2015), 'Emotional reaction facilitates the brain and behavioural impact of graphic cigarette warning labels in smokers', Tobacco control, 24(3): 225-232.

Wang, A.-L., K. Ruparel, J. W. Loughead, A. A. Strasser, S. J. Blady, K. G. Lynch, D. Romer, J. N. Cappella, C. Lerman and D. D. Langleben (2013), 'Content Matters: Neuroimaging Investigation of Brain and Behavioral Impact of Televised Anti-Tobacco Public Service Announcements', The Journal of neuroscience: the official journal of the Society for Neuroscience, 33(17): 7420-7427. 
Warren, C. W., L. Riley, S. Asma, M. P. Eriksen, L. Green, C. Blanton, C. Loo, S. Batchelor and D. Yach (2000), 'Tobacco use by youth: a surveillance report from the Global Youth Tobacco Survey project', Bulletin of the World Health Organization, 78(7): 868-876.

Weber, R., R. Huskey, J. M. Mangus, A. Westcott-Baker and B. O. Turner (2015), 'Neural Predictors of Message Effectiveness during Counterarguing in Antidrug Campaigns', Communication Monographs, 82(1): 4-30.

Welborn, B. L., M. D. Lieberman, D. Goldenberg, A. J. Fuligni, A. Galvan and E. H. Telzer (2016), 'Neural Mechanisms of Social Influence in Adolescence', Social Cognitive and Affective Neuroscience, 11(1): 100-109.

Williams, A. F. (2003), 'Teenage drivers: patterns of risk', Journal of safety research, 34(1): 5-15.

Zimmerman, R. S., R. L. Donohew, P. Palmgreen, S. Noar, P. K. Cupp and B. Floyd (2011), 'Designing Media and Classroom Interventions Targeting High Sensation Seeking or Impulsive Adolescents to Prevent Drug Abuse and Risky Sexual Behavior', in Inbibitory Control and Drug Abuse Prevention, New York, NY: Springer, 263-280.

Zimring, F. E. (1998), American Youth Violence, Oxford, New York: Oxford University Press. 